\title{
The use of biomarkers for the etiologic diagnosis of MCI in Europe: An EADC survey
}

Martina Bocchetta ${ }^{\mathrm{a}, \mathrm{b}}$, Samantha Galluzzi ${ }^{\mathrm{a}}$, Patrick Gavin Kehoe ${ }^{\mathrm{c}}$, Eduardo Aguera ${ }^{\mathrm{d}}$,
Roberto Bernabei $^{\mathrm{e}}$, Roger Bullock ${ }^{\mathrm{f}}$, Mathieu Ceccaldi ${ }^{\mathrm{g}}$, Jean-François Dartigues ${ }^{\mathrm{h}}$, Alexandre de Mendonça ${ }^{\mathrm{i}}$, Mira Didic ${ }^{\mathrm{g}}$, Maria Eriksdotter ${ }^{\mathrm{j}}$, Olivier Félician ${ }^{\mathrm{g}}$, Lutz Frölich ${ }^{\mathrm{k}}$, Hermann-Josef Gertz ${ }^{1}$, Merja Hallikainen ${ }^{\mathrm{m}}$, Steen G. Hasselbalch ${ }^{\mathrm{n}}$, Lucrezia Hausner ${ }^{\mathrm{k}}$, Isabell Heuser $^{\circ}$, Frank Jessen ${ }^{\mathrm{p}, \mathrm{q}}$, Roy W. Jones ${ }^{\mathrm{r}}$, Alexander Kurz ${ }^{\mathrm{s}}$, Brian Lawlor ${ }^{\mathrm{t}}$, Alberto Lleo ${ }^{\mathrm{u}}$, Pablo Martinez-Lage ${ }^{\mathrm{V}}$, Patrizia Mecocci ${ }^{\mathrm{W}}$, Shima Mehrabian ${ }^{\mathrm{x}}$, Andreas Monsch ${ }^{\mathrm{y}}$, Flavio Nobili ${ }^{\mathrm{z}}$, Agneta Nordberg ${ }^{j}$, Marcel Olde Rikkert ${ }^{\mathrm{aa}}$, Jean-Marc Orgogozo ${ }^{\mathrm{h}}$, Florence Pasquier ${ }^{\text {bb }}$, Oliver Peters $^{\mathrm{cc}}$, Eric Salmon ${ }^{\mathrm{dd}}$, Carmen Sánchez-Castellano ${ }^{\mathrm{ee}}$, Isabel Santana ${ }^{\mathrm{ff}}$, Marie Sarazin ${ }^{\mathrm{gg}}$, Latchezar Traykov $^{\mathrm{x}}$, Magda Tsolaki ${ }^{\mathrm{hh}}$, Pieter Jelle Visser ${ }^{\mathrm{ii}, j \mathrm{j}}$, ̊̊sa K. Wallin ${ }^{\mathrm{kk}}$, Gordon Wilcock ${ }^{11}$, David Wilkinson $^{\mathrm{mm}}$, Henrike Wolf ${ }^{\mathrm{q}, \mathrm{nn}}$, Görsev Yener $^{\mathrm{oo}}$, Dina Zekry ${ }^{\mathrm{pp}}$, Giovanni B. Frisoni ${ }^{\mathrm{a}, \mathrm{qq}}$,*

${ }^{a}$ LENITEM (Laboratory of Epidemiology, Neuroimaging and Telemedicine), IRCCS Istituto Centro S. Giovanni di Dio Fatebenefratelli, Brescia, Italy

${ }^{b}$ Department of Molecular and Translational Medicine, University of Brescia, Brescia, Italy

${ }^{c}$ Dementia Research Group, School of Clinical Sciences, University of Bristol, Frenchay Hospital, Bristol, UK

${ }^{d}$ Servicio Neurologia, Hospital Universitario Reina Sofía Córdoba, Spain

${ }^{e}$ Department of Gerontological, Geriatric and Psychiatric Sciences, Università Cattolica del Sacro Cuore, Rome, Italy

${ }^{f}$ Victoria Centre, Swindon, UK

${ }^{g}$ Service de Neurologie et Neuropsychologie, CHU Timone and INSERM U1106, Aix-Marseille Univ, Marseille, France

${ }^{\text {h}}$ Bordeaux CMRR, France

${ }^{i}$ Laboratory of Neurosciences, Inst of Molecular Medicine, Lisbon, Portugal

${ }^{j}$ Department of Neurobiology, Care Sciences and Society, Karolinska Institutet, Stockholm, Sweden

${ }^{k}$ Department of Geriatric Psychiatry, Zentralinstitut für Seelische, Gesundheit Mannheim, University of Heidelberg, Mannheim, Germany

${ }^{l}$ Department of Psychiatry and Psychotherapy, University Hospital Leipzig, Leipzig, Germany

${ }^{m}$ University of Eastern Finland, Univ Hospital, Kuopio, Finland

${ }^{n}$ Memory Disorders Research Unit, Rigshospitalet, Copenhagen, Denmark

${ }^{o}$ Klinik und Poliklinik für Psychiatrie und Psychotherapie, Universitätsklinikum Bonn, Bonn, Germany

${ }^{p}$ Department of Psychiatry, University of Bonn, Bonn, Germany

${ }^{q}$ German Center for Neurodegenerative Disease (DZNE), Bonn, Germany

${ }^{r}$ RICE - The Research Institute for the Care of Older People, Royal United Hospital, Bath, UK

${ }^{s}$ Technische Universität Psychiatrische Klinik, Munchen, Germany

${ }^{t}$ Mercer's Institue for Research on Ageing, St James' Hospital, Dublin, Ireland

${ }^{u}$ Memory Unit, Neurology Service, Hospital Santa Creu i, Sant Pau, Barcelona, Spain

${ }^{v}$ Fundacion CITA-Alzheimer Fundazioa, San Sebastian, Spain

${ }^{w}$ Section of Gerontology and Geriatrics, University of Perugia, Perugia, Italy

${ }^{x}$ Department of Neurology, Univ Hospital Alexandrovska, Sofia, Bulgaria

${ }^{y}$ Memory Clinic, University Center for Medicine of Aging Basel, Felix Platter Hospital, Basel, Switzerland

${ }^{z}$ Clinical Neurology, Dept of Neuroscience (DINOGMI), University of Genoa, Genoa, Italy

${ }^{a}$ Department of Geriatric Medicine, Radboud University Medical Centre, Radboud Alzheimer Centre, Nijmegen, Netherlands

${ }^{b b} \mathrm{CHU}$ de Lille, Université de Lille 2, Lille, France

${ }^{c c}$ Department of Psychiatry, Charité-Universitätsmedizin Berlin, Berlin, Germany

${ }^{d d}$ Université de Liège, Cyclotron Research Centre, Liege, Belgium 


\author{
${ }^{e e}$ Servicio de Geriatría, Hospital Universitario Ramón y Cajal, Madrid, Spain \\ ${ }^{f f}$ Neurology Department, Coimbra University Hospital, Coimbra, Portugal \\ ${ }^{g g}$ Neurologie de la Mémoire et du Langage, Université Paris Descartes, Sorbonne Paris Cité, INSERM UMR S894, Centre Hospitalier Sainte Anne, \\ Paris, France \\ ${ }^{h h} 3 r d$ Department of Neurology, Aristotle University of Thessaloniki, Thessaloniki, Greece \\ ${ }^{i i}$ Alzheimer Centre, Vrije Univ Medical Centre, Amsterdam, Netherlands \\ ${ }^{i j}$ Alzheimer centre Maastricht University, Maastricht, Netherlands \\ ${ }^{k}$ Clinical Memory Research Unit, Lund University, Memory Clinic Malmö, Sweden

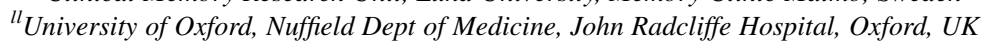 \\ ${ }^{m m}$ Memory Assessment and Research Centre MARC, Moorgreen Hospital, Southampton, UK \\ ${ }^{n n}$ Department of Psychiatry Research, Zurich, Switzerland \\ ${ }^{\circ o}$ Dokuz Eylül University, Izmir, Turkey \\ ${ }^{p p}$ Department of Internal Medicine and Geriatrics, University Hospitals and University of Geneva, Geneva, Switzerland \\ ${ }^{q q}$ Memory Clinic and Laboratoire de Neuroimagerie du Vieillissement (LANVIE), University Hospitals and University of Geneva, Geneva, Switzerland
}

\begin{abstract}
We investigated the use of Alzheimer's disease (AD) biomarkers in European Alzheimer's Disease Consortium centers and assessed their perceived usefulness for the etiologic diagnosis of mild cognitive impairment (MCI). We surveyed availability, frequency of use, and confidence in diagnostic usefulness of markers of brain amyloidosis (amyloid positron emission tomography [PET], cerebrospinal fluid $[\mathrm{CSF}] \mathrm{A} \beta 42$ ) and neurodegeneration (medial temporal atrophy [MTA] on MR, fluorodeoxyglucose positron emission tomography [FDG-PET], CSF tau). The most frequently used biomarker is visually rated MTA (75\% of the 37 responders reported using it "always/ frequently") followed by CSF markers (22\%), FDG-PET (16\%), and amyloid-PET (3\%). Only $45 \%$ of responders perceive MTA as contributing to diagnostic confidence, where the contribution was rated as "moderate". Seventy-nine percent of responders felt "very/extremely" comfortable delivering a diagnosis of MCI due to AD when both amyloid and neuronal injury biomarkers were abnormal $(P<.02$ versus any individual biomarker). Responders largely agreed that a combination of amyloidosis and neuronal injury biomarkers was a strongly indicative AD signature.
\end{abstract}

(C) 2014 The Alzheimer's Association. All rights reserved.

Keywords: $\quad$ Biomarkers; Alzheimer's disease; Mild cognitive impairment; Diagnosis

\section{Introduction}

The International Working Group [1,2] and National Institute on Aging-Alzheimer's Association criteria [3-5] recognize the importance of imaging and cerebrospinal fluid (CSF) markers for the early diagnosis of Alzheimer's disease (AD) at the stage of mild cognitive impairment (MCI). The proposed criteria state that positivity on one or more biomarkers of brain amyloidosis (decreased levels of amyloid beta 42 (A $\beta 42)$ in the CSF and increased binding of amyloid imaging ligands on positron emission tomography [PET]) and neuronal injury (medial temporal atrophy [MTA] on magnetic resonance imaging (MRI), increased total tau or phospho-tau in the CSF, and cortical temporoparietal, and posterior cingulate cortex hypometabolism on fluorodeoxyglucose positron emission tomography [FDG-PET] or if FDG-PET is unavailable, hypoperfusion on single positron emission computed tomography [SPECT]) is associated with a high probability that the patient's cognitive impairment is due to AD pathology.

The authors of the revised criteria [1-5] are cautious regarding the use of biomarkers in the clinical practice. They advise that these should be regarded as "research" criteria predominantly, although they may be applicable in some specialized clinical services with appropriate knowledge and facilities. The revised criteria were developed on the basis of scientific evidence from the literature, although they still have limitations being based on available studies, such as study design heterogeneity (i.e. population, follow-up time, cut-off and normative values, and lack of Standard Operating Procedures [SOPs] for biomarker assessment) [6]. Importantly, the criteria still need to be properly validated, implying that a large body of empirical evidence still needs to be collected to show that, when applied following appropriate SOPs, the proposed combinations of biomarkers enhance diagnostic accuracy.

Some structured programs are currently ongoing where MCI patients are assessed by traditional clinical assessment supported by an extended range of core biomarkers that include MTA, CSFA $\beta /$ tau, and hypometabolism on FDG-PET [7-10]. However, these programs are constrained to a limited number of academic memory clinics, whereas the measurement of individual biomarkers are now available to and used by a much larger number of memory clinics with a clinical research background, including those of the European Alzheimer's Disease Consortium (EADC, http://www.eadc. info). While validation of the revised diagnostic criteria is still awaited and large cases series allowing the empirical head-to-head study of the diagnostic usefulness of biomarkers 
are still pending, this study investigated the perception among leading dementia researchers of diagnostic usefulness in patients with MCI assessed in the memory clinics of the EADC centers with a suspected dementing disorder. This study has been conceived and carried out by the Disease Markers Special Interest Group of the EADC.

\section{Methods}

The EADC is a network of 63 European centers of clinical and biomedical research excellence working in the field of $\mathrm{AD}$ (http://www.eadc.info/sito/pagine/home.php). It aims at increasing the scientific understanding of $\mathrm{AD}$ and at developing therapy for $\mathrm{AD}$ symptoms.

An online questionnaire (12 items, see Appendix) assessed: (i) clinical duties of responders (six items); (ii) availability and frequency of use of biomarker examinations (i.e. MR, FDG-PET, amyloid-PET, CSF measurements) in patients with MCI (four items); (iii) the incremental diagnostic value of biomarkers (one item), and (iv) diagnostic confidence for the diagnosis of prodromal $\mathrm{AD}$ or MCI due to $\mathrm{AD}$ (clinical vignette, one item). The questionnaire was made accessible to EADC memory clinics from November 8 to December 10, 2012. Items 1 to 6 regarded the responder's details about his or her specialty, institution, country, role in the EADC memory clinic and number of new MCI patients consulted in a typical month. Item 7 investigated the frequency of use of the biomarkers; items 8 and 10 investigated whether the biomarkers are used to support the diagnosis and which of them are included into the clinical report; whereas item 9 asked to specify the kind of tool/scale used for the assessment of imaging markers. Item 11 asked to rate on a five-point Likert scale the additional diagnostic value of each biomarker on top of clinical and magnetic resonance assessment; item 12 presented a clinical vignette and asked to rate the diagnostic confidence on a five-point Likert scale, on the basis of abnormality of the biomarkers (alone or combined).

Responders were required to be clinically qualified psychologists/physicians who are actively involved in the clinical management and diagnosis of patients. Only one responder was allowed per centre. When more than one completed questionnaire was received from a centre, answers were compounded by the coordinators of this study at IRCCS Fatebenefratelli in Brescia, and the result was validated with the site PI.

Table 1 summarizes the examinations, biomarkers, and pertinent reading tools investigated in this study. It should be noted that what is referred to as "traditional read" for structural MR and FDG-PET differed from the "structured visual assessment" for the amyloid-PET. The former refers to the visual assessment made by a radiologist who based his or her interpretation using a reference of normality developed on the basis of his or her own experience. The latter refers to a structured assessment, for which image interpretation is based on detailed, clear, and specific instruction [11].
Table 1

Examination, biomarker, and pertinent reading tools in this study

\begin{tabular}{|c|c|c|}
\hline Examination & Biomarker & Reading tool \\
\hline Structural MR & $\begin{array}{l}\text { Medial temporal } \\
\text { atrophy (MTA) }\end{array}$ & $\begin{array}{l}\text { Traditional read } \\
\text { Visual Rating Scale [12] } \\
\text { Manual hippocampal } \\
\quad \text { volumetry }\end{array}$ \\
\hline FDG-PET & $\begin{array}{l}\text { Temporoparietal }(\mathrm{TP}) \\
\text { and posterior cingulate } \\
\text { (PC) hypometabolism }\end{array}$ & $\begin{array}{l}\text { Traditional read } \\
\text { Statistical maps }[48,49] \\
\text { Scalar metrics }[39,40]\end{array}$ \\
\hline CSF & $\begin{array}{l}\mathrm{A} \beta 40, \mathrm{~A} \beta 42, \mathrm{~A} \beta 42: \mathrm{A} \beta 40, \\
\quad \text { tau, phospho-tau }\end{array}$ & ELISA [50] \\
\hline Amyloid-PET & Cortical amyloid uptake & $\begin{array}{l}\text { Statistical maps [48] } \\
\text { Structured visual } \\
\text { assessment }[11]\end{array}$ \\
\hline
\end{tabular}

Abbreviations: MR, magnetic resonance; FDG, fluorodeoxyglucose; PET, positron emission tomography; CSF, cerebrospinal fluid.

NOTE. Traditional read is subjective expert judgment based on unstructured visual assessment of black and white or colored maps. The structured visual assessment refers to the interpretation of the image based on detailed, clear and specific instruction. Statistical maps refer to a parametric z-score image, derived by comparing each image on a pixel-by-pixel basis with a normative reference database. Scalar metrics refer to an automated summary measure of Alzheimer's disease (AD)-related hypometabolism based on the comparison of individual images with a normative reference data set in a predefined $\mathrm{AD}$ mask.

The regional division of Europe is based on United Nation definition (http://unstats.un.org/unsd/methods/m49/ m49regin.htm\#europe) and the estimated population of each Country is based on http://epp.eurostat.ec.europa.eu/ portal/page/portal/eurostat/home/, as of 01/01/2012.

Answers to the questionnaires were analyzed using SPSS software version 12.0 (SPSS Inc., Chicago, IL, USA). We used Mann-Whitney non-parametric U-test to compare ordinal scores between groups of responders (i.e. those who "never" or "rarely" use the biomarker and those who "regularly", "frequently", or "always" use it). We used Spearman rank correlation to measure the strength of the association of diagnostic confidence and judgment of additional diagnostic value with the actual frequency of biomarker use and features of the responders (age, sex, and years of clinical experience with AD). The level of significance was set at $P<.05$.

\section{Results}

\subsection{Responders' features}

Thirty-seven out of 63 EADC centers participated in our survey (participation rate 59\%). Figure 1 shows the geographic distribution of responding and non-responding centers. Most responders were located in Western (41\%), Southern (32\%), and Northern Europe (24\%). Only one center was located in Eastern Europe (3\%). As expected, the number of responder centers in a country correlated with its estimated population (Spearman's $\rho=.920, P<.005$ ), under the hypothesis that if there is a bigger population then there will be more centers by demand of the population and thus likely more to respond. 


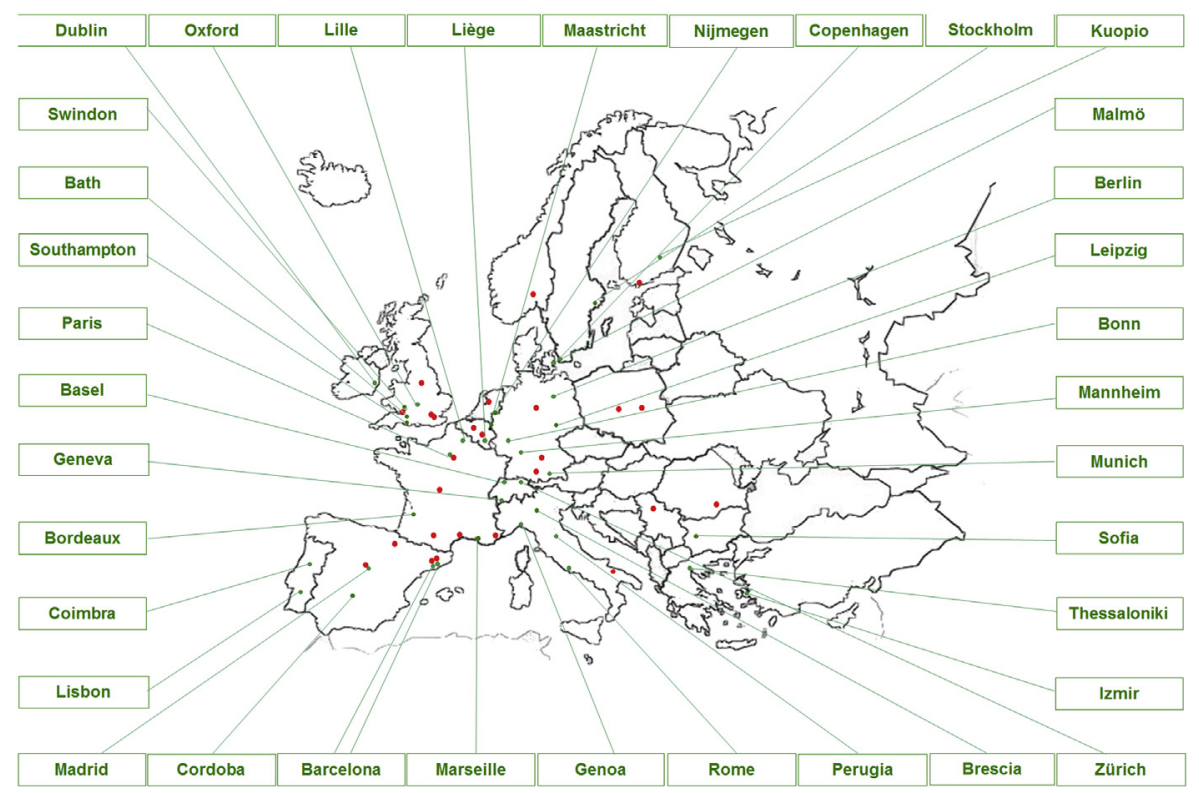

Fig. 1. Geographical distribution of the 37 European Alzheimer's Disease Consortium (EADC) centers taking part to this study (in green). In red the EADC centers that did not fill in the questionnaire: Belgium (Antwerp, Leuven); Finland (Helsinki); France (Montpellier, Nice, Paris, Toulouse, Tours); Germany (Erlangen, Goettingen, Ulm); Italy (Bari); Norway (Oslo); Poland (Lodz, Warsaw); Romania (Bucharest); Serbia (Belgrade); Spain (Barcelona, Barcelona, Madrid, Pamplona); The Netherlands (Amsterdam); United Kingdom (Bristol, London, London, Manchester).

Responders' specialties were Neurology (46\%), Geriatrics $(22 \%)$, Psychiatry (16\%), Geriatric Psychiatry (11\%), and others (Neuropsychiatry/Neuropsychology) (5\%). About two thirds of responders $(65 \%)$ were the lead of a memory clinic with administrative duties, $16 \%$ were leads without administrative duties, and $19 \%$ worked as staff members.

\subsection{Clinical role}

All responders were involved in the delivery of clinical consultations to persons with MCI. In a typical month, the largest number of responders (43\%) usually consulted up to 10 new patients with MCI; 38\% 11-20 new patients, $14 \%$ 21-40 new patients, and only $2(5 \%)$ more than 40 new patients.

\subsection{Use of biomarkers and reading tools}

\subsubsection{Imaging biomarkers}

All responders (100\%) had access to (i.e. patients could potentially undergo) structural MR, 78\% FDG-PET, and $38 \%$ amyloid-PET. Thirty-four responders (92\%) used imaging to support their etiological diagnosis of MCI, and 33 (89\%) included the results of the marker assessment into the clinical report for the patient or the primary care physician. Table 2 shows imaging examinations and reading tools that were typically included in clinical reports. Considering all reported examinations, the most frequently used tools were visual rating for MR (61\%) (i.e. Scheltens's MTA scale [12]); the traditional read for FDG-PET (70\%); and structured visual assessment for amyloid-PET (80\%) [11]. Eigh- teen percent $(6 / 33)$ of responders rated cerebrovascular disease on MR with a number of different structured visual scales such as Fazekas's [13], Wahlund's [14], and Pasquier's [15].

Five responders reported the use of perfusion SPECT, which was assessed with the traditional visual read by four

Table 2

Imaging examinations and reading tools used for the assessment of patients with MCI in EADC centers

\begin{tabular}{llllr}
\hline $\begin{array}{l}\text { Imaging } \\
\text { examination }\end{array}$ & $\begin{array}{l}\text { Clinical } \\
\text { report }\end{array}$ & Reading tool & n/total & $\%$ \\
\hline Structural MR & Not included & & $4 / 37$ & 11 \\
& included & & $33 / 37$ & 89 \\
& & Visual Rating Scale [12] & $20 / 33$ & 61 \\
& & Manual hippocampal & $1 / 33$ & 3 \\
& & volumetry & & \\
FDG-PET & Not included & Only traditional read & $12 / 33$ & 36 \\
& included & & $9 / 29$ & 31 \\
& & Statistical maps [48,49] & $20 / 29$ & 69 \\
& & Scalar metrics [39,40] & $2 / 20$ & 20 \\
& & Only traditional read & $14 / 20$ & 70 \\
Amyloid-PET & Not included & & $9 / 14$ & 64 \\
& included & & $5 / 14$ & 36 \\
& & Statistical maps [49] & $1 / 5$ & 20 \\
& & Structured visual & $4 / 5$ & 80 \\
& & assessment [11] & & \\
\hline
\end{tabular}

Abbreviations: MR, magnetic resonance; FDG, fluorodeoxyglucose; PET, positron emission tomography.

NOTE. The denominator is the number of centers that used the biomarker disaggregated by inclusion of the biomarker result in the clinical report. More than one reading tool was accepted. 
and with Statistical Parametric Mapping by one. Three responders reported the use of computed tomography-no specific reading tool or scale was reported.

All of the 33 clinicians who included, if performed, at least one imaging marker into the clinical report, included MR: $27 \%$ of them included MR alone, but the largest proportion (39\%) included MR together with FDG-PET. Only five centers $(15 \%)$ included all neuroimaging examinations (i.e. MR, FDG-PET or SPECT, and amyloid-PET).

\subsubsection{CSF Biomarkers and genotype}

Thirty-one responders (84\%) used CSF markers and 21 (68\%) of them included the CSF results in the clinical report. Of these 21, 19 (91\%) included A $\beta 42,19$ (91\%) included total tau and phospho-tau (that were always measured together), and in $33 \%$ of these centers tau markers were measured combined with $\mathrm{A} \beta 42$, whereas eight (38\%) included the ratio $A \beta 42: A \beta 40$, whereas only six (29\%) included $A \beta 40$. Eight centers (38\%) included APOE genotype. Only two centers $(10 \%)$ included all six measures (A $\beta 42, A \beta 40, A \beta 42: A \beta 40$, phospho-tau, total tau, and APOE genotype) in the clinical report.

\subsection{Frequency of biomarker use}

Figure 2 shows that $62 \%$ of responders always used MR (i.e. for more than $80 \%$ of patients), which is the same percentage of responders who never used amyloid-PET. Fortynine and $40 \%$ of responders respectively only rarely used FDG-PET and CSF biomarkers (i.e. in less than $10 \%$ of patients). Structural MR was prescribed for almost all MCI patients; lumbar puncture and CSF biomarker assessment were the second most prescribed procedure (84\%), followed by FDG-PET (78\%) (Figure 2). The distributions of frequency for MR and amyloid-PET were significantly different from the ones of any other biomarker $(P<.001$, on MannWhitney U-test), whereas CSF did not significantly differ from FDG-PET $(P=.281)$.

\subsubsection{Combinations of biomarkers}

We found that 13 centers $(35 \%)$ assessed all four core biomarkers: MTA on MR (TP-PCC hypometabolism or hypoperfusion on FDG-PET OR SPECT), CSF A $\beta /$ tau, and cortical uptake on amyloid-PET. Eighteen centers (49\%) assessed at least one neuronal injury (MTA on MR [TP-PCC hypometabolism or hypoperfusion on FDG-PET OR SPECT] or CSF tau) and one amyloidosis biomarker (CSF A $\beta$ or cortical uptake on amyloid-PET); four (11\%) assessed only neuronal injury biomarkers; and two (5\%) assessed only the structural biomarker (MTA on MR).

\subsection{Incremental diagnostic value}

Figure 3 shows responders' judgments of the diagnostic value of biomarkers in addition to clinical and MR assessment. Sixty percent of responders believed that CSF biomarkers have "great" to "decisive" incremental diagnostic value, while the respective figures were $43 \%$ and $46 \%$ for amyloid-PET and FDG-PET. The distributions of the ordinal scores of judgment were not significantly different (for all pairs of biomarker comparison, $P \geq .21$ on Mann-Whitney U-test).

If responders are divided up according to frequency of biomarker use ("never or rarely" vs. "regularly, frequently or always", see Figure 2) responders who routinely used FDG-PET regarded this biomarker had greater added value than those who used it less often $(P=.009$ on MannWhitney U-test). The frequency of CSF biomarker or amyloid-PET use did not affect the judgment of usefulness ( $P=.11$ and $P=.75$ on Mann-Whitney U-test). These results were confirmed in a correlation analysis where frequency of biomarker use and judgment of diagnostic usefulness were treated as ordinal variables (FDG-PET: Spearman's $\rho=.457, P=.004$; CSF A $\beta /$ tau: $\rho=.272$, $P=.104$; amyloid-PET: $\rho=.147, P=.385$ ). We have also investigated the diagnostic confidence of amyloidPET in those responders who frequently used CSF and

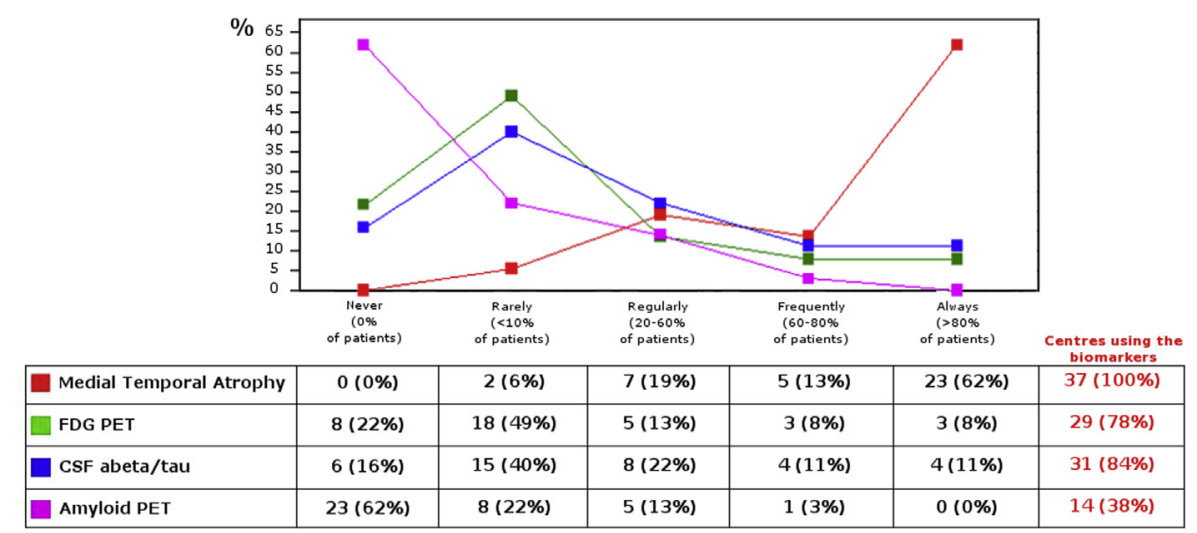

Fig. 2. Frequency of biomarker use in the assessment of patients with mild cognitive impairment in European Alzheimer's Disease Consortium (EADC) centers. Values are presented as \% and number of the 37 participating EADC centers. \% reported in the graph corresponds to the one reported in the cells. Column headings of the table correspond to x-axis points on the graph. FDG, fluorodeoxyglucose; PET, positron emission tomography; CSF, cerebrospinal fluid. 

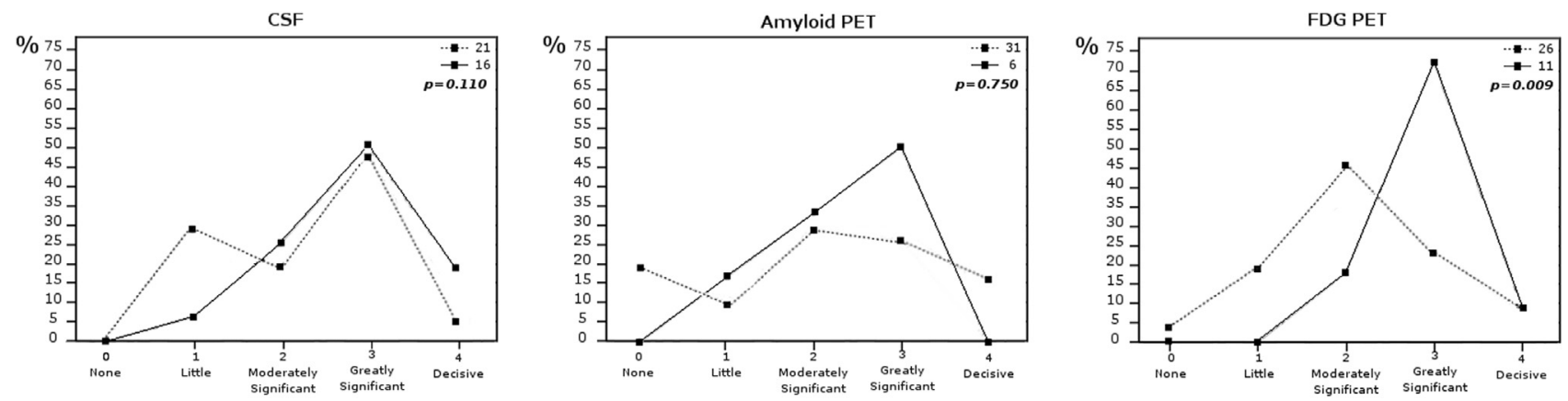

Fig. 3. European Alzheimer's Disease Consortium (EADC) centers' judgment of the incremental diagnostic value of biomarkers in the assessment of mild cognitive impairment patients on top of clinical assessment and magnetic resonance imaging. The black dotted line denotes those centers who never or rarely use the biomarker, while the black straight line those who used regularly, frequently or always (see Fig. 2). The number of responding centers is reported in the upper right corner of each graph. The ordinal score distribution of the two groups is significantly different only for FDG-PET, with frequent users assigning greater diagnostic value. CSF, cerebrospinal fluid; PET, positron emission tomography; FDG, fluorodeoxyglucose.

vice versa, but no significant relationships were found (see Supplementary Material, Figure S1).

We did not find any significant relationship between the incremental diagnostic value of FDG-PET and reading procedure (traditional reading and quantitative tools; Spearman's $\rho=.197, P=.405$ ), nor of reading tools between the groups who "never or rarely used" and those who "routinely used" ( $P=.503$ on Mann-Whitney U-test). When considering only centers that routinely used the pertinent biomarkers (black straight line), the modal value was "Greatly Significant" for all biomarkers (50\% for CSF and for amyloid, and 73\% of centers for FDG-PET). We found that for centers that did not assess routinely amyloid and FDG-PET (black dotted line), the modal value for these two markers was "Moderately Significant", thus lower. Moreover, amyloid and FDG-PET were considered as not adding any diagnostic value by $19 \%$ and $4 \%$ of clinicians, respectively, who did not frequently use them $(P=.848$ on Mann-Whitney U-test). Moreover no significant correlations between incremental diagnostic value and age, sex or years of clinical experience with $\mathrm{AD}$ of responders were found (see Table S1, Supplementary Material).

\subsection{Diagnostic confidence (clinical vignette)}

Table 3 indicates that confidence with MCI diagnosis on the basis of MTA alone was the lowest $(P=.019$ vs. TPPC hypometabolism alone, $P=.01$ vs. abnormal CSF alone, $P=.026$ vs. abnormal amyloid-PET alone, and $P<.00001$ vs. the combination of one amyloid marker and one neuronal injury marker). Diagnostic confidence was not different among abnormal FDG-PET, CSF A $\beta /$ tau, and amyloidPET biomarkers considered alone, but it was significantly lower than the combination of amyloid and neuronal injury markers $(P=.004, P=.008$, and $P=.016$, on MannWhitney U-test).

We did not find any significant correlation between diagnostic confidence and the frequency of biomarker use for any of the biomarkers (MTA on MR: $\rho=.077, P=.655$; TP-PC hypometabolism on FDG-PET: $\rho=.140, P=.533$; abnormal CSF A $\beta /$ tau: $\rho=-.078, P=.724$, abnormal amyloid-PET: $\rho=.076, P=.872$, on Spearman test). Moreover no significant correlations between diagnostic confidence and age, sex, or years of clinical experience of responders were found (see Table S2, Supplementary Material).

\section{Discussion}

This was a survey of EADC centers on the reported use and perceptions of usefulness of $\mathrm{AD}$ biomarkers for the etiological diagnosis of MCI. The centers included in this survey currently use some biomarkers for the etiological diagnosis of MCI. Responders largely agreed that a combination of amyloidosis and neuronal injury biomarkers was the most convincing in vivo signature of $\mathrm{AD}$. Interestingly, it seems that EADC centers were pretty confident in the newer biomarkers (CSF and amyloid-PET) than in the most frequently used biomarker (MTA).

Consistent with common practice, among the 37 responding centers structural MR was the most frequently used technical examination in the evaluation of MCI patients, but it also was the one perceived to contribute the least to diagnostic confidence. This apparent contradiction can be explained by structural imaging being regarded as mandatory at least once in the assessment of patients with cognitive impairment, with the aim to exclude intracranial pathology $[16,17]$. Moreover, MR is widely available and non-invasive, and thus practical. The poor contribution to diagnostic confidence might be due to MR being usually rated with traditional or visual approaches, inherently open to subjective interpretation, only one centre used the more accurate fully quantitative hippocampal volumetry. However, the time and effort required is not trivial, accounting for its exceptional clinical use. The future availability of well-validated automated methods might help the dissemination of this biomarker to the clinical routine. Alternatively, because MTA becomes abnormal late in the disease process [18], it may lack 
Table 3

Clinical vignette: physicians' diagnostic confidence. Values are presented as \% of responders ( $P$-value denotes significance at Mann-Whitney U-test)

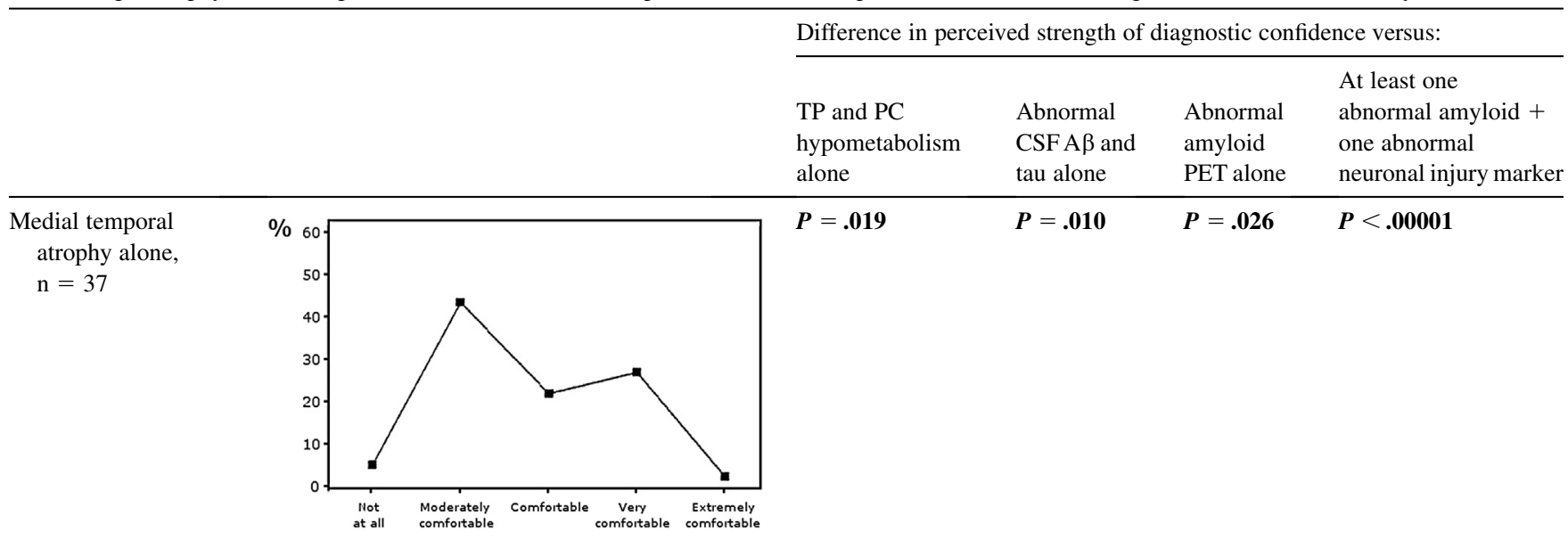

TP and PC

hypometabolism alone, $\mathrm{n}=32$

Abnormal CSF A $\beta$ and tau alone, $\mathrm{n}=35$

$\%$

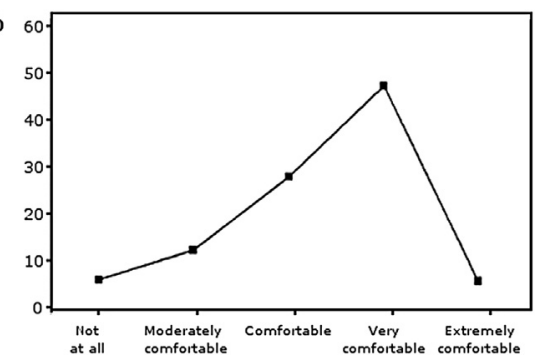

$\%$

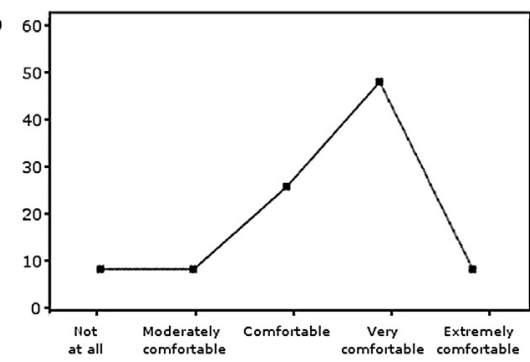

Abnormal amyloid PET alone, $\mathrm{n}=26$

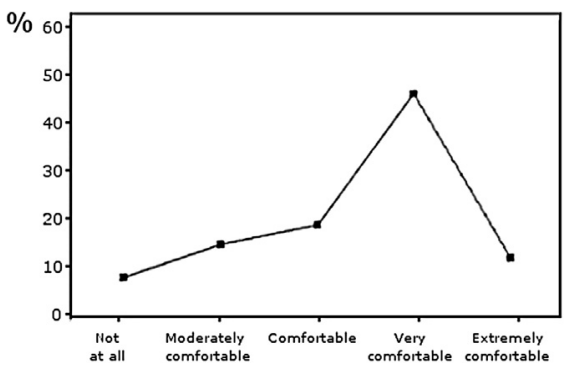

$$
P=.737 \quad P=.771 \quad P=.004
$$


Table 3

Clinical vignette: physicians' diagnostic confidence. Values are presented as \% of responders (P-value denotes significance at Mann-Whitney U-test) (Continued)

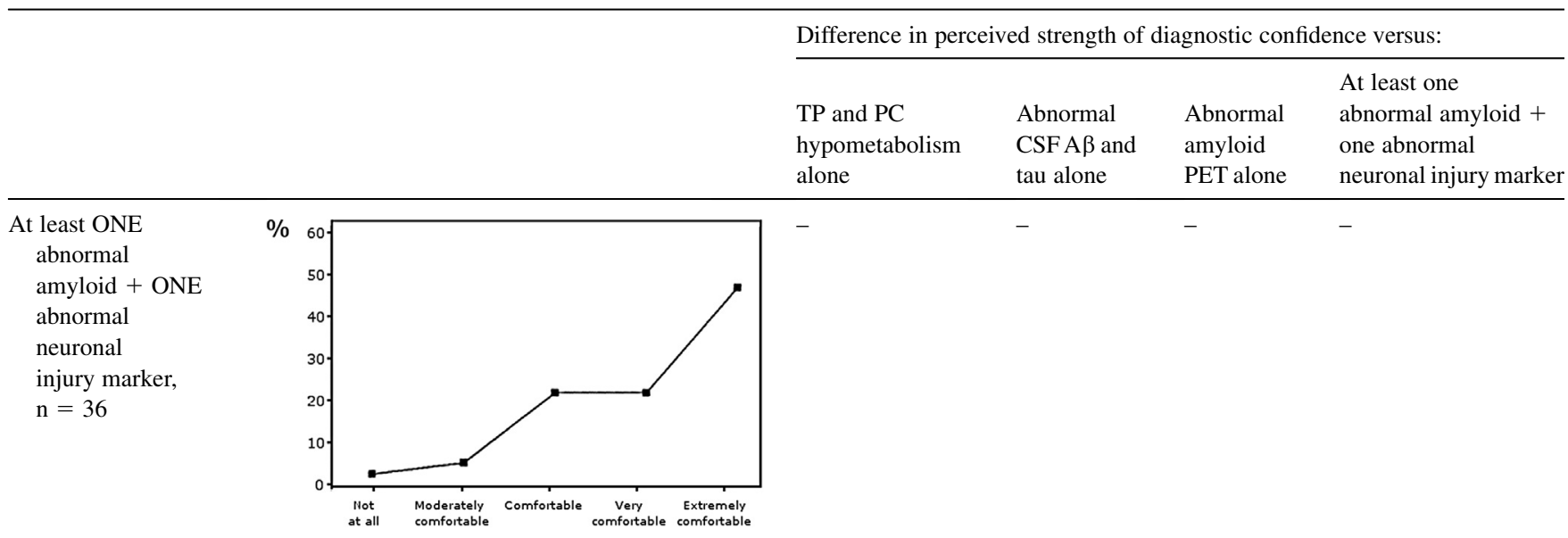

Abbreviations: TP, temporoparietal; PC, posterior cingulate; CSF, cerebrospinal fluidx; PET, positron emission tomography.

sensitivity at the MCI stage. Last, hippocampal atrophy is known to lack specificity, as it can be present in non-AD forms of dementia, like vascular dementia [19,20], semantic dementia [21], Parkinson's dementia [20], and frontotemporal lobar degeneration [22]. Interestingly, EADC centers seem to endorse the notion that the amyloid cascade stays at the core of the pathophysiology of AD.

Among responding centers, CSF biomarkers are much less frequently used than MR, consistently with the relatively poor acceptance of lumbar puncture by patients in many European countries. This study extends and confirms the data about the CSF use reported by a former survey among EADC centers [23]. However, the participants of the present study perceived CSF assays as having greatly significant incremental diagnostic value over MR and clinical assessment. This was the opinion regardless of actual use, and the impact on confidence was significant even when used in isolation. This is in line with the literature, showing that CSF $\mathrm{A} \beta 42$ or $\mathrm{A} \beta 42 / \mathrm{A} \beta 40$ ratio in combination with total tau and phospho-tau may be useful to identify $\mathrm{AD}$ with sensitivity and specificity values up to $95 \%$ [24]. The pattern of low $A \beta 42$ and high total tau and phospho-tau has been referred to as the "AD signature" [25-27].

By analogy to CSF biomarkers, hypometabolism on FDG-PET was also less frequently used than MR, which is understandable in view of its relatively high costs (i.e. 1000-2000 euros per scan). An interesting finding is that the judgment of the incremental diagnostic value of FDGPET varied according to the frequency of its use. Responders who routinely used FDG-PET assigned greater incremental diagnostic value than non-users - an added value that was perceived greater than that of CSF biomarkers. Although the association was not affected by the reading procedure ("traditional visual reading" or "quantitative tool"), a considerable amount of evidence indicates that automated quantitative tools are more accurate to detect the $\mathrm{AD}$ pattern of cortical hypometabolism than traditional readouts [28-30] and attenuate the "beginner effect" [29]. Another possible explanation is that FDG-PET requires experience to be of value, in contrast to CSF, which uses very simple cut-off values.

Not surprisingly, amyloid-PET was the least popular examination among responding centers, due to its still low availability in clinical centers, low specificity and high associated costs. Amyloid tracers were recently approved by both FDA and EMA [31-33] for use in diagnostic assessment, thus their use in the clinic will probably increase. In agreement with the literature, showing that amyloid-PET provides similar or complementary information as CSF A $\beta 42$ assays [34,35], the perception of added diagnostic value of responders was similar to the latter. However, whether amyloidPET and CSF A $\beta 42$ assays provide redundant or complementary information, is an issue under active investigation.

Among responding centers, the most widely used reading tool for all imaging biomarkers was structured or unstructured visual readouts. Although this is inevitable in the case of amyloid imaging where the only FDA- and EMA-approved procedure is structured visual rating, a number of automated or semiautomated tools are available for structural MR and FDG-PET [36-40]. However, despite their demonstrated superiority over visual assessments [28-30,41,42], these more quantitative measures are used by a minority of centers. We argue that scientific societies should engage in an active campaign to promote their use, once properly validated, 
in cognitively impaired patients undergoing assessment for suspected AD.

A sizable proportion (about one third) of responding centers was using all core AD biomarkers, and about half used at least one biomarker of neuronal injury and one of amyloidosis. Unsurprisingly, in the clinical vignette the highest diagnostic confidence was reported when biomarkers were available in this combination. What is notable, however, is the huge gain of confidence from individual to combined biomarker assessments. This is in line with published evidence, e.g. the assessment of a panel of biomarkers combined instead of a biomarker alone (whatever it is) drastically increases the diagnostic accuracy in MCI and $\mathrm{AD}$ patients [7-10,24,43].

This study has some limitations. First, the participation of EADC centers to the survey was incomplete. At the time of this survey, the EADC numbered 63 centers, and of these 37 (59\%) took part to the survey. Although this proportion is low by epidemiologic standards and is consistent with a potential selection bias, it must be highlighted that even the denominator of the proportion is not rigorously defined. In fact, participation to the EADC rests on a set of rather relaxed rules including submitting a request to the Steering Committee, where entry is subject to a centre delivering clinical dementia care but is also involved in clinical research and taking part in regular EADC semiannual meetings. Thus, even the denominator is affected by selection bias, supporting the notion that the 37 participating centers are an ultraselected group more likely to use biomarkers in clinical routine than nonresponding centers. What they likely share is comparable levels of academic interest in research, and cultural or operational interest in biomarkers for diagnosis, which might have prompted their participation. Moreover, it should be acknowledged a potential site bias: it cannot be excluded that the 37 responder centers are those more enthusiastic on biomarkers, more active users, more interested and therefore more likely to respond to a survey about these markers than non-responder centers. However, given the current hot debate on early diagnosis and the polarization of the field into opposing ideological positions [44], a selection bias in favor of sites strongly against biomarkers cannot be ruled out. In addition, it should be noticed that EADC centers have been actively involved for more than 10 years in clinical and/or preclinical dementia research and clinical trials. It follows that the conclusions of this survey may not be generalizable to any memory clinic with a focus on routine medical care.

Another study limitation is that here we provided a clinical vignette with clear-cut positive results, but it would be interesting also to investigate how often clinicians encounter negative, ambiguous or disparate results for different biomarkers and how they interpret these results. Further surveys can be launched to assess these is- sues, which would be informative and critical in terms of clinical applicability of these examinations. Moreover, we have not addressed the impact on diagnostic confidence of important modifiers such as age at onset of cognitive disturbances, APOE genotype and co-morbidities, factors that can alter the certainty of the diagnosis. It is known that early onset patients with dementia (before age 65) have greater change of biomarkers than late onset patients $[45,46]$.

Here we only investigated the reported use of biomarkers in the context of MCI evaluation, and we did not collect data on the number of actually performed examinations. Indeed, in this survey all quantitative data (numbers and percentages) are based on the 37 responding sites. Even if it might be objected that this questionnaire yields subjective opinions and findings and not objective evidence on diagnostic value, it must be remarked that currently there is no high quality data available empirically comparing and combining the different biomarkers for MCI. These empirical data showing the added value of the use of biomarkers for early diagnosis are urgently needed, together with empirical evidence of early diagnostic disclosure for positive results and a cost-benefit evaluation of the implementation of these examinations in clinical practice. As a consequence, we need guidelines on how and when individual biomarkers (or a combination of them) should and should not be used as they represent unnecessary costs, based on demographic, clinical, logistical and economical variables. The process of shared decision making [47] might also be considered as relevant in using biomarkers for the diagnosis of MCI due to AD. Last, the current survey was based on visually rating of amyloid-PET; we anticipate that quantitative measures of ligand uptake will be increasingly used in the coming years.

In conclusion, these results suggest that biomarkers for the etiological diagnosis of MCI are currently used in a sizable group of selected European memory clinics, and not only for research purposes. These academic centers represent a forerunner community of early users who will soon benefit from current efforts to develop and validate SOPs for biomarker collection and measurement. However, to be implemented in the clinical practice for the etiologic diagnosis of MCI, the biomarkers should be definitely and properly validated and guidelines for clinical standard of care should be developed.

\section{Acknowledgments}

The authors would like to thank the EADC centers taking part to this survey: IRCCS-Istituto Centro San Giovanni di Dio-Fatebenefratelli, Brescia, ITA (PI Giovanni B Frisoni); Servicio Neurologia, Hospital universitario Reina Sofía Córdoba, ESP (PI Fernando Sanchez-Lopez); Department of Gerontological, Geriatric and Psychiatric 
Sciences, Università Cattolica del Sacro Cuore, Rome, ITA (PI Roberto Bernabei); Victoria Centre, Swindon, UK (PI Roger Bullock); Service de Neurologi et Neuropsychologie, CHU Timone, Marseille, FRA (PI Mira Didic); Bordeaux CMRR, FRA (PI Jean-Marc Orgogozo); Lab of Neurosciences, Inst of Molecular Medicine, Lisbon, POR (PI Alexandre de Mendonça); Department of Neurobiology, Care Sciences and Society, Karolinska Institutet, Stockholm, SWE (PI Bengt Winblad); Department of Geriatric Psychiatry, Zentralinstitutfür Seelische, Gesundheit Mannheim, Univ of Heidelberg, Mannheim, GER (PI Lutz Frölich); Department of Psychiatry and Psychotherapy, University Hospital Leipzig, Leipzig, GER (PI Hermann-Josef Gertz); University of Kuopio, Kuopio Univ Hospital, Kuopio, FIN (PI Hilkka Soininen); Memory Disorders Research Unit, Copenhagen, DEN (PI Gunhild Waldemar); Department of Psychiatry, CharitéUniversitätsmedizin Berlin, Berlin, GER (PI Isabell Heuser); Klinik und Poliklinikfür Psychiatrie und Psychotherapie, Universitätsklinikum Bonn, Bonn, GER (PI Wolfgang Meyer); RICE - The Research Inst for the Care of Older People, Royal United Hospital, Bath, UK (PI Roy W Jones); Technische Universität Psychiatrische Klinik, Munchen, GER (PI Alexander Kurz); Mercer's Inst for Research on Ageing, St James' Hospital, Dublin, IRL (PI Brian Lawlor); Memory Unit, Neurology Service, Hospital Santa Creu i, Sant Pau, Barcelona, ESP (PI Teresa Gomez-Isla); Fundacio ACE, Inst Català de Neurociencies Aplicades, Barcelona, ESP (PI Mercè BoadaRovira); University of Perugia, Ospedale S. Maria della Misericordia, Perugia, ITA (PI Patrizia Mecocci); Department of Neurology, Univ Hospital Alexandrovska, Sofia, BUL (PI Latchezar Traykov); Memory clinic, Department of Geriatrics, University Hospital Basel, CH (PI Andreas Monsch); University of Genoa, Genoa, ITA (PI Guido Rodriguez); Radboud Alzheimer Centre, University Hospital Nijmegen, Department of Geriatric Medicine, Nijmegen, NED (PI Marcel Olde Rikkert); CHU de Lille, Université de Lille 2, Lille, FRA (PI Florence Pasquier); Université de Liège, Cyclotron Research Centre, Liege, BEL (PI Eric Salmon); Servicio de Geriatría, Hospital Universitario Ramón y Cajal, Madrid, ESP (PI Alfonso Cruz); Neurology Department, Coimbra University Hospital, Coimbra, POR (PI Isabel Santana); Centre de Neuropsychologie, Pavillon Paul Castaigne, Hôpital Salpétrière, Paris, FRA (PI Bruno Dubois);Aristotle University of Thessaloniki, Thessaloniki, GRE (PI Magda Tsolaki); Alzheimer Centre, Vrije Univ Medical Centre; Amsterdam, NED (PI Philip Scheltens); Memory clinic Malmò, SWE (PI Lennart Minthon); University of Oxford, Nuffield Dept of Medicine, John Radcliffe Hospital, Oxford, UK (PI Gordon Wilcock); Memory Assessment and Research Centre MARC, Moorgreen Hospital, Southampton, UK (PI David Wilkinson); Department of Psychiatry Research, Zurich, CH (PI Christoph Hock); Dokuz Eylül University, Izmir, TUR (PI GörsevYener); Department of Internal Medicine and Geriatrics, University Hospitals and University of Geneva, Geneva, CH (PI Dina Zekry).

\section{Supplementary data}

Supplementary data related to this article can be found at http://dx.doi.org/10.1016/j.jalz.2014.06.006.

\section{RESEARCH IN CONTEXT}

1. Systematic review: While validation of the revised diagnostic criteria for Alzheimer's disease (AD) is still awaited and large cases series allowing the empirical head-to-head study of the diagnostic usefulness of $\mathrm{AD}$ biomarkers are still pending, this study investigated the perception among European Alzheimer's Disease Consortium centers of diagnostic usefulness of biomarkers in patients with mild cognitive impairment (MCI).

2. Interpretation: Responder centers currently use the core biomarkers (cerebrospinal fluid A $\beta /$ tau levels, medial temporal atrophy, hypometabolism on fluorodeoxyglucose positron emission tomography, FDGPET) for the etiological diagnosis of MCI and largely agreed that a combination of amyloidosis and neuronal injury biomarkers was a strongly indicative AD signature.

3. Future directions: The use of biomarkers for the etiological diagnosis of MCI is widespread in the European academic research memory clinics surveyed here. They represent a forerunner community of early users who will soon benefit from current efforts to develop and validate standard operating procedures for biomarker collection and measurement.

\section{References}

[1] Dubois B, Feldman HH, Jacova C, Dekosky ST, Barberger-Gateau P, Cummings J, et al. Research criteria for the diagnosis of Alzheimer's disease: revising the NINCDS-ADRDA criteria. Lancet Neurol 2007; 6:734-46.

[2] Dubois B, Feldman HH, Jacova C, Cummings JL, Dekosky ST, Barberger-Gateau P, et al. Revising the definition of Alzheimer's disease: a new lexicon. Lancet Neurol 2010;9:1118-27.

[3] Sperling RA, Aisen PS, Beckett LA, Bennett DA, Craft S, Fagan AM, et al. Toward defining the preclinical stages of Alzheimer's disease: recommendations from the national institute on aging-Alzheimer's association workgroups on diagnostic guidelines for Alzheimer's disease. Alzheimers Dement 2011;7:280-92.

[4] Albert MS, DeKosky ST, Dickson D, Dubois B, Feldman HH, Fox NC, et al. The diagnosis of mild cognitive impairment due to Alzheimer's disease: recommendations from the national institute 
on aging-Alzheimer's association workgroups on diagnostic guidelines for Alzheimer's disease. Alzheimers Dement 2011;7:270-9.

[5] McKhann GM, Knopman DS, Chertkow H, Hyman BT, Jack CR Jr, Kawas CH, et al. The diagnosis of dementia due to Alzheimer's disease: recommendations from the national institute on aging-Alzheimer's association workgroups on diagnostic guidelines for Alzheimer's disease. Alzheimers Dement 2011;7:263-9.

[6] Frisoni GB, Hampel H, O’Brien JT, Ritchie K, Winblad B. Revised criteria for Alzheimer's disease: what are the lessons for clinicians? Lancet Neurol 2011;10:598-601.

[7] Prestia A, Caroli A, Herholz K, Reiman E, Chen K, Jagust WJ, et al. Diagnostic accuracy of markers for prodromal Alzheimer's disease in independent clinical series. Alzheimers Dement 2013;9:677-86.

[8] Prestia A, Caroli A, van derFlier WM, Ossenkoppele R, Van Berckel B, Barkhof F, et al. Prediction of dementia in MCI patients based on core diagnostic markers for Alzheimer disease. Neurology 2013;80:1048-56.

[9] Shaffer JL, Petrella JR, Sheldon FC, Choudhury KR, Calhoun VD, Coleman RE, et al. Predicting cognitive decline in subjects at risk for Alzheimer disease by using combined cerebrospinal fluid, MR imaging, and PET biomarkers. Radiology 2013;266:583-91.

[10] Choo IH, Ni R, Scholl M, Wall A, Almkvist O, Nordberg A. Combination of (18)F-FDG-PET and cerebrospinal fluid biomarkers as a better predictor of the progression to Alzheimer's disease in mild cognitive impairment patients. J Alzheimers Dis 2013;33:929-39.

[11] Clark CM, Pontecorvo MJ, Beach TG, Bedell BJ, Coleman RE, Doraiswamy PM, et al. Cerebral PET with florbetapir compared with neuropathology at autopsy for detection of neuritic amyloid-beta plaques: a prospective cohort study. Lancet Neurol 2012;11:669-78.

[12] Scheltens P, Leys D, Barkhof F, Huglo D, Weinstein HC, Vermersch P, et al. Atrophy of medial temporal lobes on MRI in "probable" Alzheimer's disease and normal ageing: diagnostic value and neuropsychological correlates. J Neurol Neurosurg Psychiatry 1992;55:967-72.

[13] Fazekas F, Chawluk JB, Alavi A, Hurtig HI, Zimmerman RA. MR signal abnormalities at $1.5 \mathrm{~T}$ in Alzheimer's dementia and normal aging. AJR Am J Roentgenol 1987;149:351-6.

[14] Wahlund LO, Barkhof F, Fazekas F, Bronge L, Augustin M, Sjogren M, et al. A new rating scale for age-related white matter changes applicable to MRI and CT. Stroke 2001;32:1318-22.

[15] Pasquier F, Leys D, Weerts JG, Mounier-Vehier F, Barkhof F, Scheltens P. Inter- and intraobserver reproducibility of cerebral atrophy assessment on MRI scans with hemispheric infarcts. EurNeurol 1996;36:268-72.

[16] Hort J, O'Brien JT, Gainotti G, Pirttila T, Popescu BO, Rektorova I, et al. EFNS guidelines for the diagnosis and management of Alzheimer's disease. Eur J Neurol 2010;17:1236-48.

[17] Knopman DS, DeKosky ST, Cummings JL, Chui H, Corey-Bloom J, Relkin N, et al. Practice parameter: diagnosis of dementia (an evidence-based review). Report of the quality standards subcommittee of the American Academy of neurology. Neurology 2001;56:1143-53.

[18] Jack CR Jr, Knopman DS, Jagust WJ, Shaw LM, Aisen PS, Weiner MW, et al. Hypothetical model of dynamic biomarkers of the Alzheimer's pathological cascade. Lancet Neurol 2010;9:119-28.

[19] Bastos-Leite AJ, van der Flier WM, van Straaten EC, Staekenborg SS, Scheltens P, Barkhof F. The contribution of medial temporal lobe atrophy and vascular pathology to cognitive impairment in vascular dementia. Stroke 2007;38:3182-5.

[20] Laakso MP, Partanen K, Riekkinen P, Lehtovirta M, Helkala EL, Hallikainen M, et al. Hippocampal volumes in Alzheimer's disease, Parkinson's disease with and without dementia, and in vascular dementia: an MRI study. Neurology 1996;46:678-81.

[21] Chan D, Fox NC, Scahill RI, Crum WR, Whitwell JL, Leschziner G, et al. Patterns of temporal lobe atrophy in semantic dementia and Alzheimer's disease. Ann Neurol 2001;49:433-42.

[22] van de Pol LA, Hensel A, van der Flier WM, Visser PJ, Pijnenburg YA, Barkhof F, et al. Hippocampal atrophy on MRI in frontotemporal lobar degeneration and Alzheimer's disease. J Neurol Neurosurg Psychiatry 2006;77:439-42.

[23] Slats D, Spies PE, Sjögren MJ, Visser PJ, Verbeek MM, OldeRikkert MG, et al. CSF biomarker utilisation and ethical considerations of biomarker assisted diagnosis and research in dementia: perspectives from within the European Alzheimer's Disease Consortium (EADC). J Neurol Neurosurg Psychiatry 2010; $81: 124-5$.

[24] Lista S, Garaci FG, Ewers M, Teipel S, Zetterberg H, Blennow K, et al. CSF Abeta1-42 combined with neuroimaging biomarkers in the early detection, diagnosis and prediction of Alzheimer's disease. Alzheimers Dement 2014;10:381-92.

[25] Mattsson N, Zetterberg H, Hansson O, Andreasen N, Parnetti L, Jonsson M, et al. CSF biomarkers and incipient Alzheimer disease in patients with mild cognitive impairment. JAMA 2009;302:385-93.

[26] Mitchell AJ, Monge-Argiles JA, Sanchez-Paya J. Do CSF biomarkers help clinicians predict the progression of mild cognitive impairment to dementia? PractNeurol 2010;10:202-7.

[27] Hansson O, Zetterberg H, Buchhave P, Londos E, Blennow K, Minthon L. Association between CSF biomarkers and incipient Alzheimer's disease in patients with mild cognitive impairment: a follow-up study. Lancet Neurol 2006;5:228-34.

[28] Lehman VT, Carter RE, Claassen DO, Murphy RC, Lowe V, Petersen RC, et al. Visual assessment versus quantitative threedimensional stereotactic surface projection fluorodeoxyglucose positron emission tomography for detection of mild cognitive impairment and Alzheimer disease. Clin Nucl Med 2012;37:721-6.

[29] Burdette JH, Minoshima S, Vander Borght T, Tran DD, Kuhl DE. Alzheimer disease: improved visual interpretation of PET images by using three-dimensional stereotaxic surface projections. Radiology 1996; 198:837-43.

[30] Ng S, Villemagne VL, Berlangieri S, Lee ST, Cherk M, Gong SJ, et al. Visual assessment versus quantitative assessment of 11C-PIB PET and 18F-FDG-PET for detection of Alzheimer's disease. J Nucl Med 2007; 48:547-52.

[31] Committee for Medicinal Products for Human Use (CHMP). Amyvidflorbetapir (18F). EMA/696925/2012. 2013.

[32] Food and Drug Administration. FDA approves imaging drug Amyvid. 2012. Available at: http://www.fda.gov/newsevents/newsroom/ pressannouncements/ucm299678.htm. Accessed July 22, 2014.

[33] Food and Drug Administration. FDA approves second brain imaging drug to help evaluate patients for Alzheimer's disease, dementia. 2013. Available at: http://www.fda.gov/newsevents/newsroom/ pressannouncements/ucm372261.htm. Accessed July 22, 2014.

[34] Landau SM, Lu M, Joshi AD, Pontecorvo M, Mintun MA, Trojanowski JQ, et al. Comparing PET imaging and CSF measurements of A $\beta$. Ann Neurol 2013;74:826-36.

[35] Fagan AM, Mintun MA, Mach RH, Lee SY, Dence CS, Shah AR, et al. Inverse relation between in vivo amyloid imaging load and cerebrospinal fluid Abeta42 in humans. Ann Neurol 2006;59:512-9.

[36] Morra JH, Tu Z, Apostolova LG, Green AE, Avedissian C, Madsen SK, et al. Validation of a fully automated 3D hippocampal segmentation method using subjects with Alzheimer's disease mild cognitive impairment, and elderly controls. Neuroimage 2008;43:59-68.

[37] Fischl B, Salat DH, Busa E, Albert M, Dieterich M, Haselgrove C, et al. Whole brain segmentation: automated labeling of neuroanatomical structures in the human brain. Neuron 2002;33:341-55.

[38] Wolz R, Aljabar P, Hajnal JV, Hammers A, Rueckert D. Alzheimer's Disease Neuroimaging Initiative. LEAP: learning embeddings for atlas propagation. Neuroimage 2010;49:1316-25.

[39] Chen K, Ayutyanont N, Langbaum JB, Fleisher AS, Reschke C, Lee W, et al. Characterizing Alzheimer's disease using a hypometabolic convergence index. Neuroimage 2011;56:52-60.

[40] Herholz K, Salmon E, Perani D, Baron JC, Holthoff V, Frolich L, et al. Discrimination between Alzheimer dementia and controls by automated analysis of multicenter FDG-PET. Neuroimage 2002;17:302-16. 
[41] Frisoni GB, Bocchetta M, Chetelat G, Rabinovici GD, de Leon MJ, Kaye J, et al. Imaging markers for Alzheimer disease: which vs how. Neurology 2013;81:487-500.

[42] Westman E, Cavallin L, Muehlboeck JS, Zhang Y, Mecocci P, Vellas B, et al. Sensitivity and specificity of medial temporal lobe visual ratings and multivariate regional MRI classification in Alzheimer's disease. PLoS One 2011;6:e22506.

[43] Galluzzi S, Geroldi C, Ghidoni R, Paghera B, Amicucci G, Bonetti M, et al. The new Alzheimer's criteria in a naturalistic series of patients with mild cognitive impairment. J Neurol 2010;257:2004-14.

[44] Le Couteur DG, Doust J, Creasey H, Brayne C. Political drive to screen for pre-dementia: not evidence based and ignores the harms of diagnosis. BMJ 2013;347:f5125.

[45] Frisoni GB, Pievani M, Testa C, Sabattoli F, Bresciani L, Bonetti M, et al. The topography of grey matter involvement in early and late onset Alzheimer's disease. Brain 2007;130:720-30.
[46] Bouwman FH, Schoonenboom NS, Verwey NA, van Elk EJ, Kok A, Blankenstein MA, et al. CSF biomarker levels in early and late onset Alzheimer's disease. Neurobiol Aging 2009;30:1895-901.

[47] Mulley AG, Trimble C, Elwyn G. Stop the silent misdiagnosis: patients' preferences matter. BMJ 2012;345:e6572.

[48] University of Washington. Neurostat. Available at: www.rad. washington.edu/research/Research/groups/nb1/neurostat-3d-ssp. Accessed July 22, 2014.

[49] Statistical Parametric Mapping. Statistical Parametric Mapping. Available at: www.fil.ion.ucl.ac.uk/spm. Accessed July 22, 2014.

[50] Olsson A, Vanderstichele H, Andreasen N, Meyer GD, Wallin A, Holmberg B, et al. Simultaneous measurement of betaamyloid(1-42), total tau, and phosphorylated tau (Thr181) in cerebrospinal fluid by the xMAP technology. Clin Chem 2005; $51: 336-45$. 

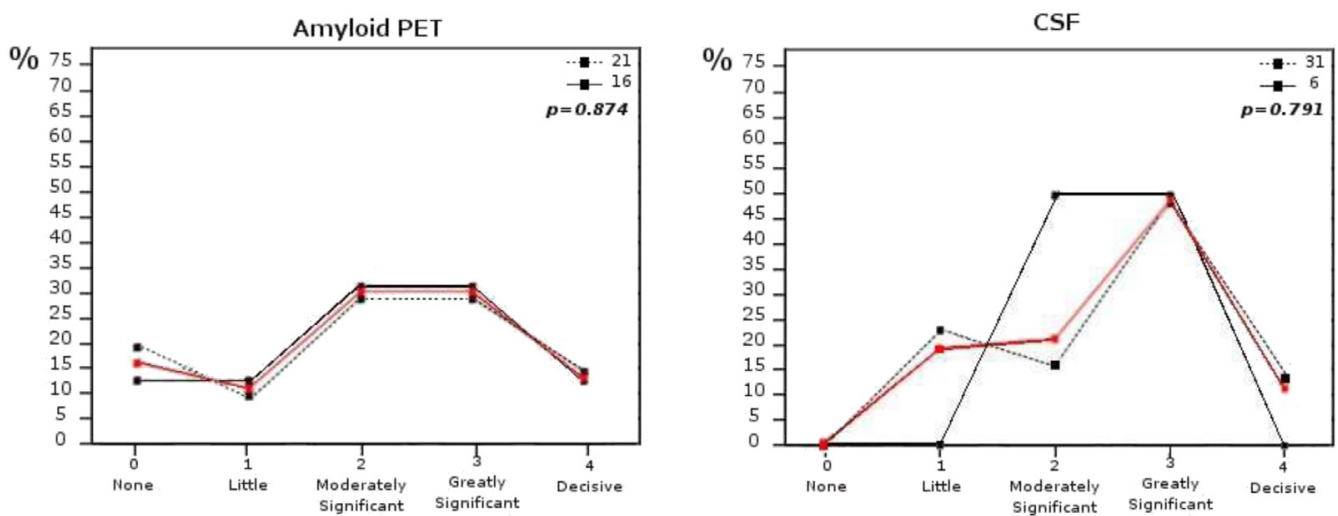

Figure S1. European Alzheimer's Disease Consortium centers' judgment of the incremental diagnostic value of amyloid-PET in those who routinely or not used the CSF (and vice versa) in the assessment of mild cognitive impairment patients on top of clinical assessment and magnetic resonance imaging. Amyloid-PET Left Panel: The black dotted line denotes those centers that never or rarely used the CSF; the black straight line those that used regularly, frequently or always the CSF. CSF - Right Panel: The black dotted line denotes those centers that never or rarely use the amyloid-PET; the black straight line those that use regularly, frequently or always the amyloid-PET. For both graphs, the red line denotes the two groups pooled together (see Figure 2). The number of responding centers is reported in the upper right corner of each graph. PET; positron emission tomography, CSF; cerebrospinal fluid.

Table S1

Correlations between incremental diagnostic value and age, sex, and years of clinical experience with $\mathrm{AD}$ of the responders ( $P$-value denotes significance on Spearman rank correlation test)

\begin{tabular}{lrrl}
\hline & & & Years of clinical \\
& Age & Sex & experience with AD \\
\hline FDG-PET & $\rho=-.132$, & $\rho=-.231$, & $\rho=-.051, P=.796$ \\
CSF & $P=.473$ & $P=.170$ & \\
Amyloid-PET & $\rho=-.032$, & $\rho=-.171$, & $\rho=-.069, P=.729$ \\
& $P=.861$ & $P=.312$ & \\
& $P=.258$ & $\rho=-.033$, & $\rho=.214, P=.275$ \\
& $P=.847$ & \\
\hline
\end{tabular}

Abbreviations: FDG; fluorodeoxyglucose, PET; positron emission tomography, CSF; cerebrospinal fluid, AD; Alzheimer's Disease.
Table S2

Correlations between diagnostic confidence and age, sex and years of clinical experience of the responders $(P$-value denotes significance on Spearman rank correlation test)

\begin{tabular}{|c|c|c|c|}
\hline & Age & Sex & $\begin{array}{l}\text { Years of clinical } \\
\text { experience } \\
\text { with } A D\end{array}$ \\
\hline $\begin{array}{l}\text { Medial temporal } \\
\text { atrophy ALONE }\end{array}$ & $\begin{array}{l}\rho=.169 \\
P=.354\end{array}$ & $\begin{array}{r}\rho=-.101 \\
P=.551\end{array}$ & $\begin{array}{l}\rho=.006 \\
P=.977\end{array}$ \\
\hline $\begin{array}{l}\text { TP and PC } \\
\text { hypo-metabolism } \\
\text { ALONE }\end{array}$ & $\begin{array}{r}\rho=-.143 \\
P=.468\end{array}$ & $\begin{array}{l}\rho=.056 \\
P=.760\end{array}$ & $\begin{array}{r}\rho=-.185 \\
P=.387\end{array}$ \\
\hline $\begin{array}{r}\text { Abnormal CSF A } \beta \\
\text { and tau ALONE }\end{array}$ & $\begin{array}{r}\rho=-.189 \\
P=.309\end{array}$ & $\begin{array}{l}\rho=.006 \\
P=.971\end{array}$ & $\begin{array}{r}\rho=-.336 \\
P=.086\end{array}$ \\
\hline $\begin{array}{l}\text { Abnormal amyloid-PET } \\
\text { ALONE }\end{array}$ & $\begin{array}{l}\rho=.142 \\
P=.528\end{array}$ & $\begin{array}{l}\rho=.253 \\
P=.212\end{array}$ & $\begin{array}{l}\rho=.225 \\
P=.340\end{array}$ \\
\hline $\begin{array}{l}\text { At least ONE abnormal } \\
\text { amyloid + ONE } \\
\text { abnormal neuronal } \\
\text { injury marker }\end{array}$ & $\begin{array}{r}\rho=-.014 \\
P=.940\end{array}$ & $\begin{array}{c}\rho=.015 \\
P=.931\end{array}$ & $\begin{array}{r}\rho=-.048 \\
P=.810\end{array}$ \\
\hline
\end{tabular}

Abbreviations: TP; temporoparietal, PC; posterior cingulate, CSF; cerebrospinal fluid, PET; positron emission tomography, AD; Alzheimer's Disease. 